\title{
Brain metastases in patients with upper gastrointestinal cancer is associated with proximally located adenocarcinoma and lymph node metastases
}

\author{
Kazuto Harada ${ }^{1,9} \cdot$ Hyunsoo Hwang ${ }^{2} \cdot$ Xuemei Wang $^{2}$ - Ahmed Abdelhakeem ${ }^{1}$ - Masaaki Iwatsuki ${ }^{1,9}$. \\ Mariela A. Blum Murphy ${ }^{1}$. Dipen M. Maru ${ }^{3}$ - Brian Weston ${ }^{4}$. Jeffrey H. Lee ${ }^{4}$. Jane E. Rogers ${ }^{5}$. Allison Trail ${ }^{1}$. \\ Namita Shanbhag ${ }^{1} \cdot$ Meina Zhao $^{1}$. Manoop S. Bhutani ${ }^{4}$. Quynh-Nhu Nguyen ${ }^{7}$. Stephen G. Swisher ${ }^{6}$. \\ Naruhiko Ikoma ${ }^{8} \cdot$ Prajnan Das $^{7} \cdot$ Wayne L. Hofstetter ${ }^{6} \cdot$ Brian D. Badgwell $^{8} \cdot$ Jaffer A. Ajani $^{1}(\mathbb{0}$
}

Received: 17 February 2020 / Accepted: 13 April 2020 / Published online: 28 April 2020

(c) The International Gastric Cancer Association and The Japanese Gastric Cancer Association 2020

\begin{abstract}
Background As cancer patients are surviving longer, more patients manifest brain metastases (BRMs). However, the rate of BRMs from upper gastrointestinal cancer is unclear. We therefore evaluated the frequency and prognostic effect of BRMs in this setting.

Methods We analyzed records of 2348 patients who were treated between January 2002 and December 2016 for upper gastrointestinal cancer, including esophageal and gastroesophageal junction adenocarcinoma (EAC; proximal EAC, Siewert types I and II), esophageal squamous cell carcinoma (ESCC), and gastric adenocarcinoma (GAC; Siewert type III and stomach cancer) in our Gastrointestinal Medical Oncology Database. Frequency, risk factors, and survival after BRMs were evaluated. Results Of 2348 patients, 68 (2.9\%) had BRMs upon follow-up. The BRM rates were as follows: proximal EAC, 4.8\%; Siewert type I, 5.9\%; Siewert type II, 2.2\%; Siewert type III, 0.7\%; ESCC: $1.2 \%$; and stomach cancer, 0\%. Among EAC patients, Siewert type I and lymph node metastases were independent the risk factors for BRMs in the multivariable analysis. The median overall survival (OS) in the 68 patients with BRMs was only 1.16 years (95\% CI 0.78-1.61). However, OS for patients who had a solitary BRM, who had BRM but no other distant metastasis, or who underwent surgery or stereotactic radiosurgery favorable.

Conclusion Patients with proximally located adenocarcinoma, or with lymph node metastases are at a higher risk for BRMs and patients fare better after treatment of isolated BRM.
\end{abstract}

Keywords Esophageal adenocarcinoma $\cdot$ Esophageal squamous-cell carcinoma $\cdot$ Gastroesophageal junction adenocarcinoma $\cdot$ Gastric adenocarcinoma $\cdot$ Brain metastases

\section{Introduction}

Upper gastrointestinal cancers (UGC) including esophageal and gastroesophageal junction adenocarcinoma (EAC; proximal EAC, Siewert types I and II), esophageal squamous cell carcinoma (ESCC), and gastric adenocarcinoma

Electronic supplementary material The online version of this article (https://doi.org/10.1007/s10120-020-01075-3) contains supplementary material, which is available to authorized users.

Jaffer A. Ajani

jajani@mdanderson.org

Extended author information available on the last page of the article
(GAC; Siewert type III and stomach cancer) are very common worldwide [1], and have a poor prognosis [2].

Approximately 70,000 new cases of brain metastases (BRMs), were diagnosed in 2007 in the United States, which was $6 \%$ of patients with newly diagnosed invasive cancers [3]. Almost $90 \%$ of BRMs result from lung, breast, melanoma, colon, or renal cancers [3]. Because BRM is often diagnosed later in the clinical course, its incidence has increased as overall survival (OS) has lengthened in many solid tumors patients [3]. Therefore, understanding BRM incidence and prognostic implications are important. However, frequency of BRM from UGC remains unclear.

A few reports have evaluated BRM incidence in UGC. Cagney et al. used data from the Surveillance, Epidemiology, 
and End Results (SEER) program to show that $1.7 \%$ of patients with esophageal cancer and $0.6 \%$ patients with gastric cancer had BRM at diagnosis, [4] but lacked details, as SEER is a national database. Several cohort and case reports of BRMs from UGC are available [5-7], but their sample size is small to correlate with clinical features (such as location or histology). Here, we analyzed 2,348 patients with UGC to evaluate frequency, risk factors, and survival influence of BRMs.

\section{Patients and methods}

\section{Patients}

We searched our prospectively maintained databases in the Department of Gastrointestinal Medical Oncology at The University of Texas MD Anderson Cancer Center (Texas, USA) and identified 2348 patients who were treated for EAC, ESCC, or GAC between January 2002 and December 2016, and included them in this analysis. No other selection criteria were applied.

Patients had extensive baseline staging, including CT and PET studies, esophagogastroduodenoscopy with endoscopic ultrasonography, and blood tests. Routine pretreatment brain imaging was not performed unless patients describe symptoms related to central nervous system disease. Tumor staging was based on the American Joint Commission on Cancer Staging Manual (8th edition) [8]. The institutional review board approved this analysis.

\section{Treatment and follow-up strategy}

Patients were treated according to the NCCN guidelines $[2,9]$. Multidisciplinary teams of medical oncologists, thoracic surgeons, surgical oncologists, radiation oncologists, gastroenterologists, thoracic radiologists, pathologists, and supporting team personnel evaluated all patients before starting any treatment. Patients were followed at 3- to 12-month intervals for at least 5 years after treatment. When patients (many of whom lived some distance away) were followed up locally, we collected their information by letter or patient referral documents. HER2 status had been tested only in patients with adenocarcinoma who have distant metastases. Head CTs or MRIs were performed only when BRM was suspected. We designated a case as having BRM(s) when solid mass(s) were noted on imaging studies. Occasionally, biopsy was performed, but it was not necessary to diagnose BRM. Meningeal dissemination was excluded from BRMs in the absence of a solid mass. Treatment for BRM(s) was decided by the multidisciplinary teams.

\section{Statistical analysis}

Patient characteristics were summarized using descriptive statistics, by frequency (\%) for categorical variables, and by median and range for continuous variables. Comparisons between groups were conducted using Fisher's exact test for categorical variables and the Wilcoxon rank-sum test for continuous variables. OS was defined as the time interval between date of diagnosis and date of death from any cause. Patients were censored at the last follow-up if they were alive at that time. The OS probabilities were estimated using the Kaplan-Meier method; log-rank tests were used to compare OS across groups. Univariate and multivariable logistic regression models were fit to assess the association between patient characteristics and the binary outcome, BRM, where variables with significance levels less than 0.05 in univariate analysis were included in the multivariable regression model except for the baseline clinical stage. All statistical analyses were performed using R 3.5.1.

\section{Results}

\section{Characteristics of patients with BRM}

Of 2348 patients, 68 (2.9\%) were found to have BRMs. The median time interval between BRMs diagnosis and initial treatment was 1.27 years (95\% CI 1.10-1.56) in clinical Stage I-IVa patients. Of 19 clinical Stage IVb patients with BRMs, 5 were had BRMs at diagnosis. The median time interval between the BRMs diagnosis and initial treatment in 14 clinical Stage IVb patients was 1.16 years $(95 \%$ CI 0.63-2.00). Table 1 shows clinical features of these patients. Incidence of BRMs was significantly higher in patients whose primary tumor was located at proximal sites (proximal EAC, 4.8\%; Siewert type I, 5.9\%; Siewert type II, 2.2\%; Siewert type III, $0.7 \%$; ESCC, $1.2 \%$; Fig. 1). No BRM developed in the stomach cancer cohort. The incidence of BRMs for EAC (4.3\% for proximal esophagus, Siewert type I and type II) was significantly higher than for ESCC (1.2\%; $P=0.009)$. Incidence of BRMs was associated with younger age, higher $\mathrm{T}$ stage, higher $\mathrm{N}$ stage, and higher clinical stage.

In clinical Stages I-II, only 2 patients developed BRMs; one in ESCC and one in Siewert type I. In clinical Stages III-IVa, 47 patients had BRMs. The incidence of BRMs was significantly higher in patients whose primary tumor was located at proximal sites (proximal EAC, 6.7\%; Siewert type I, 7.3\%; Siewert type II, 2.0\%; Siewert type III, 1.8\%; ESCC, 0.8\%; Fig. 1). In clinical Stage IVb patients, 19 had BRMs. The incidence of BRMs was higher in patients with proximal EAC, Siewert type I, Siewert type II (Fig. 1). 
Table 1 Clinical characteristics of patients with and without brain metastasis $(n=2347)$

\begin{tabular}{|c|c|c|c|}
\hline \multirow[t]{2}{*}{ Clinical feature } & \multicolumn{2}{|l|}{ Brain metastasis } & \multirow[b]{2}{*}{$P$} \\
\hline & Positive $68(2.9 \%)$ & Negative $2280(97.1 \%)$ & \\
\hline Mean age \pm SD & $59.8 \pm 10.59$ & $62.7 \pm 11.49$ & 0.03 \\
\hline Sex & & & 0.09 \\
\hline Male & $60(3.2)$ & $1809(96.8)$ & \\
\hline Female & $8(1.7)$ & $471(98.3)$ & \\
\hline Cancer type & & & $<0.001$ \\
\hline Proximal EAC & $6(4.8)$ & $119(95.2)$ & \\
\hline Siewert type I & $44(5.9)$ & $707(94.1)$ & \\
\hline Siewert type II & $14(2.2)$ & $612(97.8)$ & \\
\hline Siewert type III & $1(0.7)$ & $152(99.3)$ & \\
\hline ESCC & $3(1.2)$ & $240(98.8)$ & \\
\hline Stomach & $0(0)$ & $450(100)$ & \\
\hline Histological Type & & & 0.20 \\
\hline Adenocarcinoma & $64(3.1)$ & $1996(96.9)$ & \\
\hline Squamous cell & $3(1.2)$ & $241(98.8)$ & \\
\hline Endocrine & $1(2.9)$ & $33(97.1)$ & \\
\hline Undetermined & $0(0)$ & $10(100)$ & \\
\hline Tumor differentiation & & & 0.23 \\
\hline Well differentiated & $0(0)$ & $26(100)$ & \\
\hline Moderately differentiated & $34(3.7)$ & $883(96.3)$ & \\
\hline Poorly differentiated & $34(2.5)$ & $1319(97.5)$ & \\
\hline Undetermined & $0(0)$ & $52(100)$ & \\
\hline Baseline $\mathrm{T}$ category & & & $<0.001$ \\
\hline $\mathrm{T} 1$ & $0(0)$ & $298(100)$ & \\
\hline $\mathrm{T} 2$ & $4(2.2)$ & $179(97.8)$ & \\
\hline $\mathrm{T} 3$ & $61(3.6)$ & $1616(96.4)$ & \\
\hline $\mathrm{T} 4$ & $2(1.2)$ & $171(98.8)$ & \\
\hline TX & $1(5.9)$ & $16(94.1)$ & \\
\hline Baseline $N$ category & & & $<0.001$ \\
\hline No & $8(0.9)$ & $930(99.1)$ & \\
\hline N1 & $35(4.4)$ & 763 (95.6) & \\
\hline $\mathrm{N} 2$ & $13(3.9)$ & $321(96.1)$ & \\
\hline N3 & $12(4.6)$ & $252(95.4)$ & \\
\hline NX & $0(0)$ & $14(100)$ & \\
\hline Baseline clinical stage & & & $<0.001$ \\
\hline I & $0(0)$ & $333(100)$ & \\
\hline II & $2(0.7)$ & $271(99.3)$ & \\
\hline III & $30(3.1)$ & 924 (96.9) & \\
\hline IVa & $17(8.7)$ & $178(91.3)$ & \\
\hline $\mathrm{IVb}$ & $19(3.2)$ & $567(96.8)$ & \\
\hline$X$ & $0(0)$ & $7(100)$ & \\
\hline
\end{tabular}

Of 551 patients who underwent HER2 testing, 85 (15.4\%) tumors were HER2 positive. Six patients $(7.1 \%)$ in the HER2 positive cohort and 27 patients $(5.8 \%)$ in the HER2 negative cohort had BRMs. Therefore, HER2 status was not associated with BRMs $(P=0.66)$.

\section{Risk factors for BRMs among patients with EAC}

Among the total 2347 patients, 1502 (64\%) were EAC patients. Clinical characteristics were summarized for these EAC patients, grouped by brain metastasis status (Table 2). 


\section{Incidence rate for brain metastasis}

\section{All Cohort}

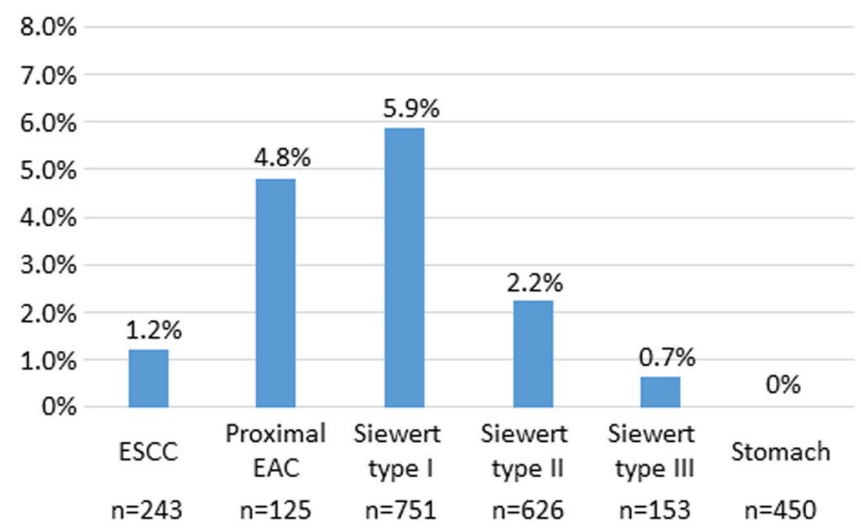

Clinical Stage III-IVa

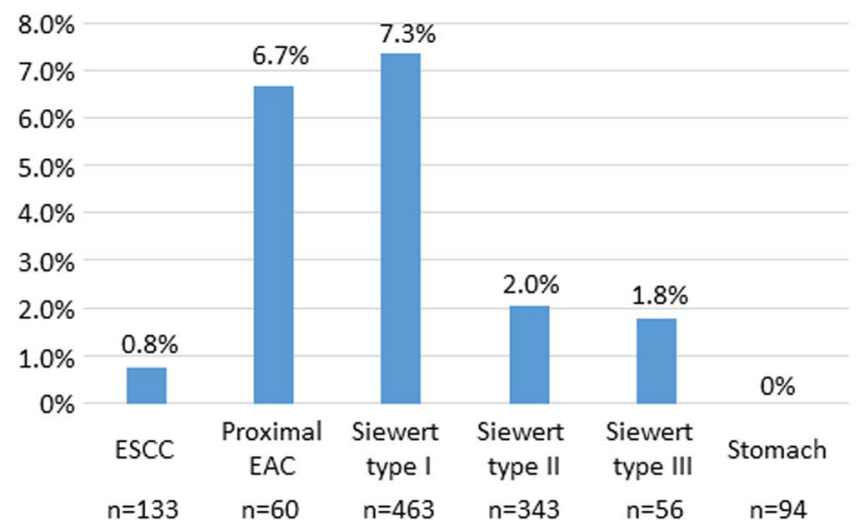

Clinical Stage I-II

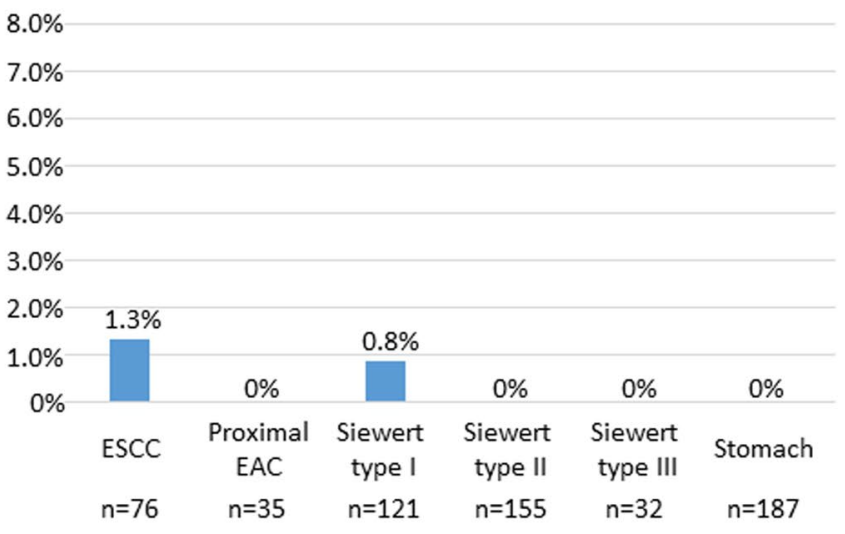

Clinical Stage IVb

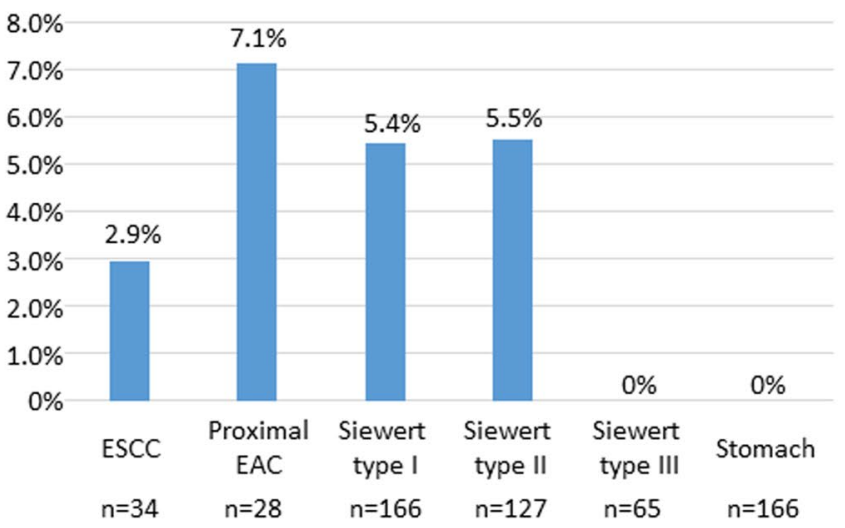

Fig. 1 Incidence rates for BRMs in all cohort and patients with clinical Stage I-II, clinical Stage III-IVa, and clinical Stage IVb

We performed univariate and multivariable logistic regression analyses to identify factors that predicts BRM in patients with EAC (Tables 3). Univariate analyses showed that younger age, location of tumor, tumor depth, lymph node metastases, and clinical stage were significantly associated with the BRMs incidence. Compared with Siewert type II, Siewert type I was a significant risk factor for BRMs (overall risk [OR]: $2.72,95 \%$ confidence interval [CI] 1.48-5.01), whereas proximal EAC tended to be a risk factor for BRMs, but not significantly so (OR: $2.20,95 \%$ CI 0.83-5.85). In multivariable analysis, tumor location and lymph node metastasis were significantly associated with BRMs (Table 3). In multivariable analysis, Siewert type I was a significant risk factor for BRM (OR: $2.44,95 \%$ CI 1.31-4.54), compared with Siewert type II, whereas proximal EAC was only marginally associated with BRMs (OR: $2.30,95 \%$ CI 0.86-6.19).

Next, we performed univariate and multivariable logistic regression analyses in clinical Stage III-IVa and clinical Stage IVb. Among 866 patients with clinical Stage III-IVa
EAC, the multivariable analysis showed that the primary tumor location and lymph node metastases were significantly associated with BRMs (Supplemental Table 1). Compared with Siewert type II, Siewert type I was a significant risk factor for BRMs (OR: 3.26, 95\% CI 1.61-8.44), whereas proximal EAC was only marginally associated with BRMs. (OR: 3.26, 95\% CI 0.92-11.6). However, among 321 patients with clinical Stage IVb, neither the primary tumor location nor lymph node metastases was not associated with BRMs (Supplemental Table 2).

\section{BRM characteristics}

Of the 68 patients with $\mathrm{BRM}(\mathrm{s}), 37$ patients had solitary BRM, 8 patients had 2 metastatic sites, and 22 patients had 3 or more BRMs. Forty-one patients had BRM(s) but no extracranial metastases, of whom 7 patients developed other extracranial metastases; thus 34 patients had only BRMs during follow-up. Twenty-seven patients had BRMs and extracranial metastases. Of the 68 patients, 31 underwent 
Table 2 Clinical characteristics of EAC patients with and without brain metastasis $(n=1502)$

\begin{tabular}{|c|c|c|c|}
\hline \multirow[t]{2}{*}{ Clinical feature } & \multicolumn{2}{|l|}{ Brain metastasis } & \multirow[b]{2}{*}{$P$} \\
\hline & Positive $64(4.3 \%)$ & Negative 1438 (96.7\%) & \\
\hline Mean age \pm SD & $59.5 \pm 10.85$ & $62.7 \pm 11.09$ & 0.03 \\
\hline Sex & & & $\geq 0.99$ \\
\hline Male & $58(4.3)$ & $1287(95.7)$ & \\
\hline Female & $6(3.8)$ & $151(96.2)$ & \\
\hline Location of tumor & & & 0.002 \\
\hline Proximal esophagus & $6(4.8)$ & $119(95.2)$ & \\
\hline Siewert type I & 44 (5.9) & $707(94.1)$ & \\
\hline Siewert type II & $14(2.2)$ & $612(97.8)$ & \\
\hline Tumor differentiation & & & 0.46 \\
\hline Well differentiated & $0(0)$ & $16(100)$ & \\
\hline Moderately differentiated & $33(5.1)$ & 618 (94.9) & \\
\hline Poorly differentiated & $31(3.9)$ & $765(96.1)$ & \\
\hline Undetermined & $0(0)$ & $39(100)$ & \\
\hline Histology & & & $\geq 0.99$ \\
\hline Adenocarcinoma & $63(4.3)$ & $1407(95.3)$ & \\
\hline Endocrine & $1(4.0)$ & $24(96.0)$ & \\
\hline N/A & $0(0)$ & $7(100)$ & \\
\hline Signet ring cell carcinoma & & & 0.86 \\
\hline Yes & $9(3.9)$ & $222(96.1)$ & \\
\hline No & $55(4.4)$ & 1206 (95.6) & \\
\hline N/A & $0(0)$ & $10(100)$ & \\
\hline Baseline $\mathrm{T}$ category & & & $<0.001$ \\
\hline $\mathrm{T} 1$ & $0(0)$ & $231(100)$ & \\
\hline $\mathrm{T} 2$ & $3(2.6)$ & $113(97.4)$ & \\
\hline $\mathrm{T} 3$ & $59(5.4)$ & $1033(94.6)$ & \\
\hline $\mathrm{T} 4$ & $1(1.9)$ & $51(98.1)$ & \\
\hline TX & $1(9.1)$ & $10(90.9)$ & \\
\hline Baseline $\mathrm{N}$ category & & & $<0.001$ \\
\hline No & $8(1.3)$ & $610(98.7)$ & \\
\hline N1 & $32(6.2)$ & $485(93.8)$ & \\
\hline $\mathrm{N} 2$ & $13(6.8)$ & $178(93.2)$ & \\
\hline N3 & $11(6.6)$ & $157(93.4)$ & \\
\hline NX & $0(0)$ & $8(100)$ & \\
\hline Baseline clinical stage & & & $<0.001$ \\
\hline I & $0(0)$ & $230(100)$ & \\
\hline IIA & $1(1.2)$ & $80(98.8)$ & \\
\hline III & $29(4.1)$ & $680(95.9)$ & \\
\hline IVA & $16(10.2)$ & $141(89.8)$ & \\
\hline IVB & $18(5.6)$ & $303(94.4)$ & \\
\hline $\mathrm{X}$ & $0(0)$ & $4(0)$ & \\
\hline
\end{tabular}

resections, 8 underwent stereotactic radiosurgery, 23 underwent whole brain radiation only, and 6 patients were lost to follow-up or had no treatment.

\section{Prognosis of patients with BRM}

Median OS in the 68 patients with BRMs was only 1.16 years after BRM diagnosis (95\% CI 0.78-1.61). Of the initial 2347 patients, 1136 developed distant metastases; 68 with BRMs and 1068 without BRM. Among the 1136 patients who had distant metastasis, OS after BRMs was significantly longer than survival after distant metastases to other organs (Median OS, 1.16 vs 0.91 years; Fig. 2a). Similarly, among the 734 EAC patients who had distant metastasis, OS after BRMs was significantly longer than 
Table 3 Univariate and multivariable logistic regression models for brain metastasis in patients with $\mathrm{EAC}(n=1502)$

\begin{tabular}{|c|c|c|c|c|}
\hline \multirow[t]{2}{*}{ Clinical feature } & \multicolumn{2}{|l|}{ Univariate } & \multicolumn{2}{|l|}{ Multivariable } \\
\hline & OR $(95 \% \mathrm{CI})$ & $P$ & OR $(95 \% \mathrm{CI})$ & $P$ \\
\hline $\mathrm{Age}^{\mathrm{a}}$ & $0.98(0.96-1.00)$ & 0.03 & $0.98(0.96-1.00)$ & 0.07 \\
\hline \multicolumn{5}{|l|}{ Sex } \\
\hline Male & 1 (reference) & & & \\
\hline Female & $0.88(0.37-2.08)$ & 0.77 & & \\
\hline \multicolumn{5}{|l|}{ Location of tumor } \\
\hline Proximal Esophagus & $2.20(0.83-5.85)$ & 0.11 & $2.30(0.86-6.19)$ & 0.09 \\
\hline Siewert type I & $2.72(1.48-5.01)$ & 0.001 & $2.44(1.31-4.54)$ & 0.005 \\
\hline Siewert type II & 1 (reference) & & 1 (reference) & \\
\hline \multicolumn{5}{|l|}{ Tumor differentiation } \\
\hline Well/moderate & 1 (reference) & & & \\
\hline Poor & $0.77(0.47-1.29)$ & 0.33 & & \\
\hline \multicolumn{5}{|c|}{ Signet ring cell carcinoma } \\
\hline No & 1 (reference) & & & \\
\hline Yes & $0.88(0.43-1.82)$ & 0.75 & & \\
\hline \multicolumn{5}{|l|}{ Baseline $\mathrm{T}$ category } \\
\hline $\mathrm{T} 1 / 2$ & 1 (reference) & & 1 (reference) & \\
\hline $\mathrm{T} 3 / \mathrm{T} 4$ & $6.34(1.98-20.4)$ & 0.0019 & $2.62(0.74-9.26)$ & 0.13 \\
\hline \multicolumn{5}{|l|}{ Baseline $\mathrm{N}$ category } \\
\hline No & 1 (reference) & & 1 (reference) & \\
\hline $\mathrm{N} 1 / \mathrm{N} 2 / \mathrm{N} 3$ & $5.20(2.46-11.0)$ & $<0.0001$ & $3.32(1.48-7.47)$ & 0.003 \\
\hline \multicolumn{5}{|l|}{ Baseline clinical stage } \\
\hline $\mathrm{I} / \mathrm{II}$ & 1 (reference) & & & \\
\hline III/IVA & $16.99(2.33-123.7)$ & 0.005 & & \\
\hline IVB & $18.41(2.44-138.8)$ & 0.005 & & \\
\hline
\end{tabular}

$C I$ confidence interval, $O R$ odds ratio

\section{Overall survival in patients with distant metastasis}

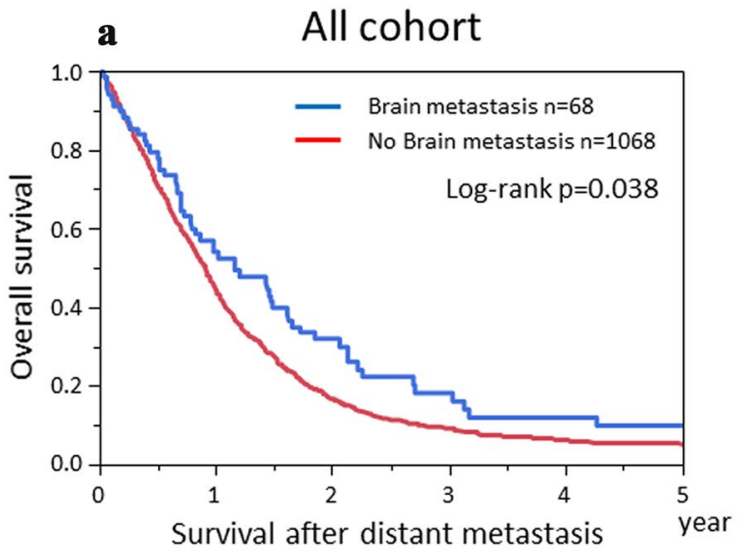

No. at risk

Brain metastasis

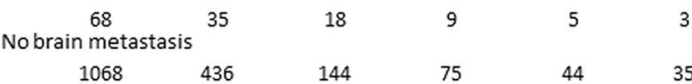

Fig. 2 Kaplan-Meier analysis of overall survival in patients with distant metastasis, categorized by presence of brain metastases. a Kaplan-Meier curves for the cohort as a whole. b Kaplan-Meier

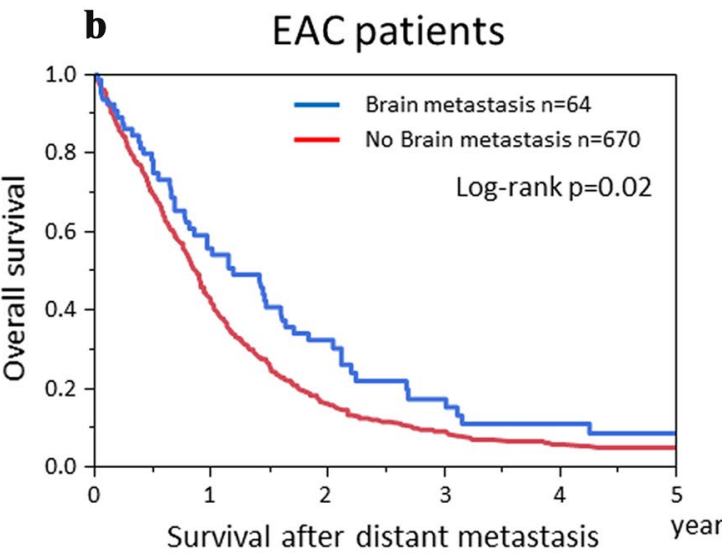

No. at risk

Brain metastasis

\begin{tabular}{|c|c|c|c|c|}
\hline No brain metastasis & 34 & 17 & 8 & 5 \\
\hline 670 & 262 & 87 & 44 & 25 \\
\hline
\end{tabular}

curves for patients with esophageal and gastroesophageal junction adenocarcinoma (EAC) 
Table 4 Brain metastases characteristics and patient survival $(n=68)$

Median survival time; years $(95 \%$

CI)

\begin{tabular}{lll} 
Number of brain meta & & \\
1 & 37 & $1.43(0.54-2.21)$ \\
2 & 8 & $0.41(0.15-1.58)$ \\
$\geq 3$ & 22 & $0.20(0.10-0.70)$ \\
N/A & 1 & - \\
Metastases pattern when diagnosed & & \\
$\quad$ Brain only & 41 & $1.20(0.61-1.48)$ \\
First metastatic site with other distant & 19 & $0.32(0.14-0.72)$ \\
$\quad$ metastasis & & \\
Metastases after other distant metastases & 8 & $0.26(0.07-0.51)$ \\
Metastasis site during follow up & & \\
Brain only & 34 & $1.09(0.50-1.58)$ \\
With lung & 9 & $0.51(0.08-1.86)$ \\
With liver & 10 & $0.22(0.08-0.32)$ \\
With bone & 14 & $0.25(0.13-0.62)$ \\
With distant lymph node & 23 & $0.27(0.18-0.62)$ \\
With others & 7 & \\
Treatment & & \\
Resection & 31 & $1.47(0.81-2.21)$ \\
Stereotactic radiosurgery & 8 & $1.12(0.38-3.11)$ \\
Whole brain radiation & 23 & $0.18(0.08-0.25)$ \\
N/A & 6 & $0.33(0.14-0.86)$ \\
\hline
\end{tabular}

OS after distant metastasis to other organs. (Median OS, 1.2 vs 0.87 years; Fig. $2 b$ ).

Median OS and 95\% confidence intervals among patients with BRM is shown in Table 4, according to several clinical strata. For patients with solitary BRM, OS was significantly longer than for patients who had multiple BRM (Fig. 3a). For patients who had BRM with no other distant metastases, OS was significantly longer than for those with extracranial metastases (Fig. 3b). For patients who underwent surgery or stereotactic radiosurgery, OS was significantly longer than for patients who underwent whole-brain radiation (Fig. 3c).

\section{Discussion}

This is the largest cohort assessing the incidence of BRMs from UGC, and it uncovered several novel findings. First, the highest risk of developing BRMs was with adenocarcinoma histology than with squamous cell carcinoma histology (EAC, $4.3 \%$; ESCC, $1.2 \%$.) and the primary tumor located more proximally from the esophagogastric junction (proximal EAC, 4.8\%; Siewert type I, 5.9\%; Siewert type II, $2.2 \%$; Siewert type III, $0.7 \%$; stomach cancer, $0 \%$ ). Second, Siewert type I and presence of lymph node metastases were risk factors for BRMs in patients with EAC. Third, patients with solitary BRM could have favorable prognoses.

We found that BRMs were more common in patients with EAC than those with GAC. Several reports have assessed BRMs from esophageal cancer. Our institute previously reported that incidence of BRM was $1.7 \%$ among 1512 patients with esophageal cancer and 3.9\% among 518 patients with esophageal cancer who received trimodal treatment $[7,10]$. Welch et al. reviewed 583 patients with esophageal cancer and identified BRMs in 22 patients (3.8\%) [6]. These incidence rates are consistent with our data (2.9\%).

We hypothesized that esophageal cancer histology types could correlate with BRMs. We showed that the incidence of BRMs from ESCC was $1.2 \%$, which is consistent with some Asian studies $[11,12]$. Ogawa et al. showed that incidence of BRMs was $1.4 \%$ among 2554 patients with ESCC [11]. Song et al. showed that BRMs incidence was 1.6\% among 1612 patients with ESCC [12]. Combined with our data, these findings show the incidence rates for BRMs from ESCC to be significantly lower than from EAC. The BRMs incidence has also been shown to vary by histology in lung cancers. Cagney et al. evaluated SEER data and showed that the incidence of BRMs from lung cancer was $14.4 \%$ a

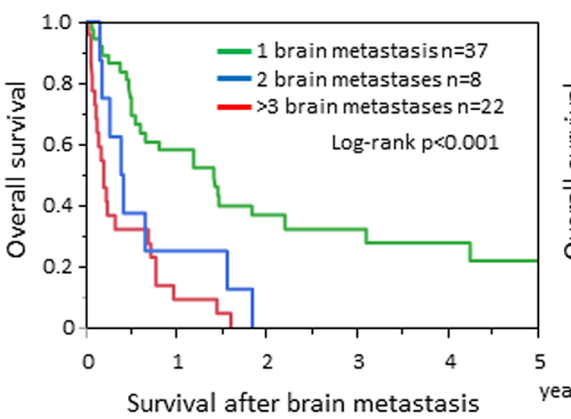

b Presence of other distant metastasis

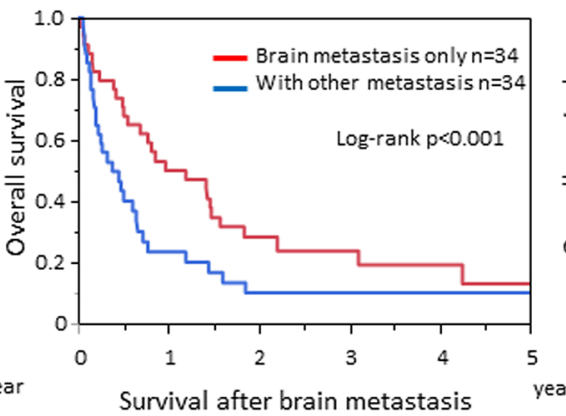

\section{Treatment for brain metastasis}

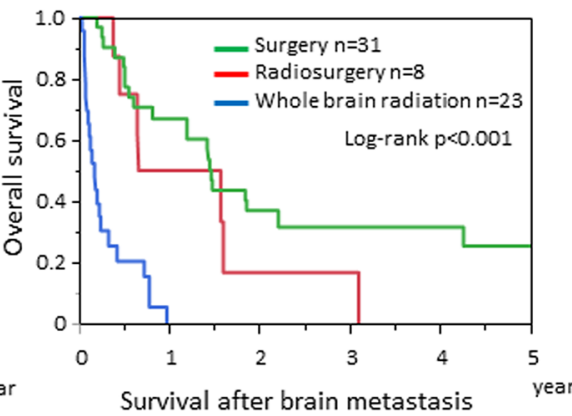

Fig. 3 Kaplan-Meier analysis of overall survival for patients with brain metastasis. a Kaplan-Meier curves by numbers of brain metastases. b Kaplan-Meier curves by presence of other distant metastases. $\mathbf{c}$ Kaplan-Meier curves by treatment for brain metastases 
from adenocarcinoma, compared with 5.3\% from squamous cell carcinoma [4]. However, why adenocarcinoma is more likely to develop BRMs is unclear.

The molecular mechanisms by which cancer cells can migrate to and grow in the brain remain unclear [13]. The molecular features of EAC and GAC overlap [14], but few GAC developed BRMs in this study. Moreover, HER2 expression have been found to be associated with BRMs $[15,16]$. However, even GAC with HER2 positive did not have higher frequency of BRMs. Microenvironment of the brain differs from that of other organs. Complexity in the brain is conferred by blood brain barrier, and microglia, or astrocytes. These findings suggest that further basic research needed to discover mechanism of BRMs.

Our data showed that median OS after BRMs was 1.16 years, which is longer than other reports about esophageal cancer (3.8-5.0 months) [4-6, 10]. Moreover, longer survival in patients with single BRM is consistent with previous reports $[5,10]$. As especially reported for lung cancer [17], our data demonstrates the contribution of resection to prolonging survival. The treatment choice for a solitary BRM is excision or stereotactic radiosurgery, whereas treatment for multiple BRMs is limited to radiation or chemotherapy only. Thus, our data indicate that identifying earlystage BRM improves chances for resection, and for improved survival.

Our study has some limitations. First, this is a retrospective study. Second, a few patients were lost to the follow-up. Third, because only patients who had symptoms of BRMs underwent evaluation, we might have missed some asymptomatic BRMs. To overcome these limitations, a prospective observational study would be ideal.

In conclusion, BRMs are rare in UGC, but patients with proximally located EAC or with lymph node metastases should be carefully monitored for BRMs.

Acknowledgements We thank Marla Brunker, from the Edanz Group (www.edanzediting.com/ac), for editing a draft of this manuscript.

Funding This research was supported by generous grants from the Caporella, Dallas, Sultan, Park, Smith, Frazier, Oaks, Vanstekelenberg, Planjery, and Cantu families, as well as from the Schecter Private Foundation, Rivercreek Foundation, Kevin Fund, Myer Fund, Dio Fund, Milrod Fund, and The University of Texas MD Anderson Cancer Center (Houston, Texas, USA) multidisciplinary grant program. This research was also supported in part by National Cancer Institute Grants CA129906, CA127672, CA138671, and CA172741; by Department of Defense Grants CA150334 and CA162445 (J.A.A.); and by a grant from the Japan Society for the Promotion of Science Overseas Research Fellowships and Program for Advancing Strategic International Networks to Accelerate the Circulation of Talented Researchers (K.H.).

\section{Compliance with ethical standards}

Conflict of interest The authors have no potential conflicts of interest to disclose.

\section{References}

1. Global Burden of Disease Cancer C, Fitzmaurice C, Allen C, Barber RM, Barregard L, Bhutta ZA, et al. Global, regional, and national cancer incidence, mortality, years of life lost, years lived with disability, and disability-adjusted life-years for 32 cancer groups, 1990 to 2015: a systematic analysis for the Global Burden of Disease study. JAMA Oncol. 2017;3:524-48.

2. Ajani JA, D’Amico TA, Almhanna K, Bentrem DJ, Besh S, Chao $\mathrm{J}$, et al. Esophageal and esophagogastric junction cancers, version 1.2015. J Natl Compr Canc Netw. 2015;13:194-227.

3. Davis FG, Dolecek TA, McCarthy BJ, Villano JL. Toward determining the lifetime occurrence of metastatic brain tumors estimated from 2007 United States cancer incidence data. Neuro Oncol. 2012;14:1171-7.

4. Cagney DN, Martin AM, Catalano PJ, Redig AJ, Lin NU, Lee EQ, et al. Incidence and prognosis of patients with brain metastases at diagnosis of systemic malignancy: a population-based study. Neuro Oncol. 2017;19(11):1511-21.

5. Kothari N, Mellon E, Hoffe SE, Frakes J, Shridhar R, Pimiento J, et al. Outcomes in patients with brain metastasis from esophageal carcinoma. J Gastrointest Oncol. 2016;7:562-9.

6. Welch G, Ross HJ, Patel NP, Jaroszewski DE, Fleischer DE, Rule WG, et al. Incidence of brain metastasis from esophageal cancer. Dis Esophagus. 2017;30:1-6.

7. Wadhwa R, Taketa T, Correa AM, Sudo K, Campagna MC, Blum $\mathrm{MA}$, et al. Incidence of brain metastases after trimodality therapy in patients with esophageal or gastroesophageal cancer: implications for screening and surveillance. Oncology. 2013;85:204-7.

8. Amin MB, Edge S, Greene F, Byrd DR, Brookland RK, Washington MK, et al. AJCC cancer staging manual. 8th ed. New York: Springer; 2017.

9. Ajani JA, D'Amico TA, Almhanna K, Bentrem DJ, Chao J, Das P, et al. Gastric cancer, version 3.2016, NCCN clinical practice guidelines in oncology. J Natl Compr Canc Netw. 2016;14:1286-312.

10. Weinberg JS, Suki D, Hanbali F, Cohen ZR, Lenzi R, Sawaya R. Metastasis of esophageal carcinoma to the brain. Cancer. 2003;98:1925-33.

11. Ogawa K, Toita T, Sueyama H, Fuwa N, Kakinohana Y, Kamata $\mathrm{M}$, et al. Brain metastases from esophageal carcinoma: natural history, prognostic factors, and outcome. Cancer. 2002;94:759-64.

12. Song Z, Lin B, Shao L, Zhang Y. Brain metastases from esophageal cancer: clinical review of 26 cases. World Neurosurg. 2014;81:131-5.

13. Fidler IJ. The biology of brain metastasis: challenges for therapy. Cancer J. 2015;21:284-93.

14. Cancer Genome Atlas Research N, Analysis Working Group, Asan U, Agency BCC, Women's H Brigham I Broad, et al. Integrated genomic characterization of oesophageal carcinoma. Nature. 2017;541:169-75.

15. Limon D, Gal O, Gordon N, Katz L, Perl G, Purim O, et al. Brain metastasis in gastroesophageal adenocarcinoma and HER2 status. J Neurooncol. 2018;138:315-20.

16. Altaha R, Crowell E, Hobbs G, Higa G, Abraham J. Increased risk of brain metastases in patients with HER-2/neu-positive breast carcinoma. Cancer. 2005; 103:442-3.

17. Patchell RA, Tibbs PA, Walsh JW, Dempsey RJ, Maruyama Y, Kryscio RJ, et al. A randomized trial of surgery in the treatment of single metastases to the brain. N Engl J Med. 1990;322:494-500.

Publisher's Note Springer Nature remains neutral with regard to jurisdictional claims in published maps and institutional affiliations. 


\section{Affiliations}

Kazuto Harada ${ }^{1,9} \cdot$ Hyunsoo Hwang ${ }^{2}$ Xuemei Wang ${ }^{2} \cdot$ Ahmed Abdelhakeem $^{1}$ - Masaaki Iwatsuki ${ }^{1,9}$. Mariela A. Blum Murphy ${ }^{1}$. Dipen M. Maru ${ }^{3}$ - Brian Weston ${ }^{4}$. Jeffrey H. Lee ${ }^{4}$. Jane E. Rogers ${ }^{5}$. Allison Trail ${ }^{1}$. Namita Shanbhag ${ }^{1}$. Meina Zhao ${ }^{1}$. Manoop S. Bhutani ${ }^{4} \cdot$ Quynh-Nhu Nguyen ${ }^{7} \cdot$ Stephen G. Swisher $^{6}$. Naruhiko Ikoma ${ }^{8} \cdot$ Prajnan Das $^{7} \cdot$ Wayne L. Hofstetter ${ }^{6} \cdot$ Brian D. Badgwell $^{8} \cdot$ Jaffer A. Ajani $^{1}$

1 Departments of Gastrointestinal Medical Oncology, The University of Texas MD Anderson Cancer Center, 1515 Holcombe Blvd, Houston, TX 77030, USA

2 Departments of Biostatistics, The University of Texas MD Anderson Cancer Center, Houston, TX 77030, USA

3 Departments of Pathology, The University of Texas MD Anderson Cancer Center, Houston, TX 77030, USA

4 Departments of Gastroenterology, The University of Texas MD Anderson Cancer Center, Houston, TX 77030, USA

5 Departments of Pharmacy Clinical Program, The University of Texas MD Anderson Cancer Center, Houston, TX 77030, USA
6 Departments of Thoracic and Cardiovascular Surgery, The University of Texas MD Anderson Cancer Center, Houston, TX 77030, USA

7 Departments of Radiation Oncology, The University of Texas MD Anderson Cancer Center, Houston, TX 77030, USA

8 Departments of Surgical Oncology, The University of Texas MD Anderson Cancer Center, Houston, TX 77030, USA

9 Department of Gastroenterological Surgery, Graduate School of Medical Science, Kumamoto University, 1-1-1 Honjo, Kumamoto 860-8556, Japan 